\title{
A modeling and simulation framework for the reliability/availability assessment of a power transmission grid subject to cascading failures under extreme weather conditions
}

\author{
Francesco Cadini ${ }^{\mathrm{a}, *}$, Gian Luca Agliardi ${ }^{\mathrm{a}}$, Enrico Zio ${ }^{\mathrm{a}, \mathrm{b}}$ \\ a Dipartimento di Energia, Politecnico di Milano, Via La Masa 34, 20156, Italy \\ ${ }^{\mathrm{b}}$ Chair System Science and the Energy Challenge, Fondation Electricite' de France (EDF), CentraleSupélec, Université Paris-Saclay, Grande Voie des Vignes, 92290 \\ Chatenay-Malabry, France
}

\section{H I G H L I G H T S}

-We combine stochastic extreme weather model and realistic power grid fault dynamics.

- We introduce a novel restoration model accounting for the weather conditions.

- The complete model is quantified by means of a customized sequential Monte Carlo.

- Reliability/availability indicators show importance of rare extreme weather events.

\section{A R T I C L E I N F O}

\section{Article history:}

Received 20 January 2016

Received in revised form 15 September 2016

Accepted 24 October 2016

Available online 7 November 2016

\section{Keywords:}

Power grids

Cascading failures

Extreme weather

Wind storm

Lightning

Monte Carlo simulation

Reliability

Availability

\begin{abstract}
A B S T R A C T
Electrical power transmission networks of many developed countries are undergoing deep transformations aimed at (i) facing the challenge offered by the stochastically fluctuating power contributions due to the continuously growing connections of renewable power generating units and (ii) decreasing their vulnerability to failures or malicious attacks and improving their resilience, in order to provide more reliable services, thus increasing both safety and profits. In this complex context, one of the major concerns is that related to the potentially catastrophic consequences of cascading failures triggered by rare and difficult to predict extreme weather events. In this work, we originally propose to combine an extreme weather stochastic model of literature to a realistic cascading failure simulator based on a direct current (DC) power flow approximation and a proportional re-dispatch strategy. The description of the dynamics of the network is completed by the introduction of a novel restoration model accounting for the operating conditions that a repair crew may encounter during an extreme weather event. The resulting model is solved by a customized sequential Monte Carlo scheme in order to quantify the impact of extreme weather events on the reliability/availability performances of the power grid. The approach is demonstrated with reference to the test case of the IEEE14 power transmission network.
\end{abstract}

(c) 2016 Elsevier Ltd. All rights reserved.

\section{Introduction}

Electric energy has an impact on all modern life activities. Consequently, the public expectation for reliable electric energy delivery has significantly grown in the past decades. For this, the so-called power grids, i.e., the interconnected systems that transmit electric power from where it is generated to where it is consumed, play a fundamental role. Thus, it comes with no surprise that the assessment of the risk of failures of these infrastructures

\footnotetext{
* Corresponding author.

E-mail address: francesco.cadini@polimi.it (F. Cadini).
}

and the study of strategies for the mitigation of the consequences have gained increased attention. However, these tasks are difficult because of the inherent complexities of the power transmission networks, the complex physical laws governing the power flow dynamics and the uncertainties affecting the boundary conditions (operational and environmental) in which they operate. Additional difficulties come from the evolution of society and energy markets, that demand changes and adaptations of the large-scale power transmission infrastructures for continuing to satisfy the requirements of reliability/availability under the continuous increase in electric power demand, avoiding congestions and overloads, so as to improve safety and increase economic margins. In addition, 
power transmission systems can no longer be considered solely as a means for energy delivery; rather, they are operated as an electricity market trading platform for shifting power volumes across different regions or even different countries, for economic reasons.

Furthermore, in recent years we have witnessed an exponential growth in the connection of renewable power generating units (photovoltaic power plants, wind farms, biomass plants, etc.) and poly-generation systems (electrical power, heat and cooling), which add even more complexity to the system. In fact, such connections were obviously not planned in the initial design of the power networks, which now need to adapt to accommodate their fluctuating power contributions, difficult to predict and geographically distributed [1].

In this new setting, concerns come for the protection from failures and attacks, and their possible cascading effects, i.e., the propagation of disconnections of components from the grid due to the power flows redistribution (manually or automatically) following the initial failure or attack. Cascading failures may lead to catastrophic disconnections of wide portions of the power transmission networks, as epitomized by several large-scale blackouts occurred in developed countries in the recent years, such as for example the blackout in Italy on September, 282003 [2], that in the United States and Canada on August 14, 2003 [3] or that in Europe on November, 62006 [4].

Normally, a power transmission network is designed and operated so that a single component disconnection does not give rise to any load shedding, so that cascading failures do not occur $(N-1$ criterion [2]); however, rare combinations of circumstances, uncommon events or inadequate countermeasures may result in further line disconnections, eventually leading to disruptive consequences. In fact, the operative conditions at a given time are determined by many, usually correlated factors, such as power demand, generating capacity, economic optimizations, technical limits and environmental conditions. Some of these factors are defined as operative limits or determined by technical-economical optimizations (e.g., the power generation) and, as such, they are more predictable. Others, such as the power demand and the environmental conditions, are uncertain by nature and many efforts are, thus, devoted to quantify the effects of these uncertainties on the power production and transportation capabilities, like, for example in [5] and [6], where the fluctuations of the power demand and of a specific environmental feature, i.e. the wind speed, are taken into account, respectively.

Furthermore, human errors, intentional attacks or extremely severe natural contingencies, such as earthquakes, volcanic eruptions and weather related events (floodings, wind storms, lightning, icing, heat and cold waves, etc.), may even directly fail the components of the network [7].

In this context, we address the problem of quantifying the reliability/availability of a transmission grid specifically with respect to the occurrence of extreme weather events, which have been identified as major threats to the operation of power networks [8]. Indeed, the decision making process of the grid designers and operators should be driven by the results of cost-benefit analyses, accounting for the reliability/availability of the system and the costs required to design, build and operate it. Proper assessment of the transmission grid reliability/availability allows informed decisions on grid design and operation by the players involved in the utility management upon. Also, reliability/availability analysis is a key factor for supporting the power quality regulation of transmission grids in many developed countries.

The problem addressed is twofold: first, a realistic, possibly stochastic, model of the extreme weather event occurrences must be developed and its parameters properly identified on the basis of available weather statistics; then, this model is coupled with realistic transmission grid dynamic models for the computation of the network performances in presence of failures triggered by the extreme weather events.

With regards to extreme weather event modeling for power grids reliability/availability estimation, the common approach is that of resorting to the "two-state" model [9], or to its improved, "three-state" version [10], where the weather conditions are divided into two or three classes, normal, adverse and, in case of three state models, major disaster, and the stochastic transitions among the different classes are modeled as homogeneous Poisson processes with constant transition rates, to be estimated from weather data.

The major drawbacks of these approaches are that (i) they are not flexible enough to represent the wide range of possible weather events types and intensity levels and (ii) their rates of occurrence are, in general, dependent on time, due to their typically seasonal behaviors. Significant advances towards a more realistic modeling of the impact of weather events on power networks reliability/availability is proposed in [11] and further extended in [16]. In those works, non-homogeneous Poisson distributions of the occurrence times and suitable distributions of the events intensities are derived on the basis of available weather statistics; furthermore, the failure rates of the networks' lines are mathematically related to the times of occurrence and the intensities of the weather events considered, following the approaches proposed in previous works of literature. In fact, it is recognized that the failure rates of the grid's components, typically taken constant, are actually significantly increased by the occurrence of severe weather events. For example, quadratic and exponential models are introduced in [12] and [13], respectively, to describe the overhead line failure rates as functions of the wind speed; a linear model is presented in [14] to model the relationship between the number of line interruptions and that of lightning flashes; the combined effect of high winds and lightning, possibly occurring during severe storms, on the grid components failure rates are analyzed in [15]. The resulting model is then solved by means of a sequential Monte Carlo scheme simulating the annual "lives" of the power grid subject to the weather events.

The approaches illustrated above, however, have been only applied either to very simple, representative power grids with few components [10] or to power distribution grids (e.g., a the Swedish rural reliability test system in [16] or an actual distribution system in the Northeast US in [17]). Limited research efforts have been made in developing ways for performing weatherrelated reliability/availability analyses on power transmission network models including realistic representations of cascading failure phenomena. For example, in [18] simple stochastic models representing the occurrences of extreme weather events are coupled to grid cascading failure models, where the network reliability is abstractly quantified with respect to the existence of connecting paths between grid nodes; however, the realistic electrical behavior of the networks are not taken into account.

In this work, we originally propose to resort to the stochastic model for representing the uncertainties related to extreme weather events introduced in [16] and further developed in [11], and to combine it to a realistic cascading failure simulation model based on a MATLAB-based DC power flow approximation of a power transmission network [19] and a proportional re-dispatch strategy [20]. Furthermore, in order to offer an even more realistic description of the power grid dynamics in response to extreme weather events, we develop an original restoration model based on the definition of suitable repair "velocities" correlated to the occurrence of the different events.

The complexity of the resulting model is such that its resolution for the estimation of the system reliability/availability indices is not easy. We, then, develop a customized, sequential Monte Carlo simulation scheme, where several annual histories of power 
transmission network lives, subject to both normal and extreme weather events-related failures, are simulated and the consequences are evaluated. The proposed modeling and simulation framework is applied for quantifying the impact of extreme weather events on a power transmission network of reference, the IEEE14 [21].

The rest of the paper is structured as follows. Section 2 describes the proposed framework for coupling the extreme weather event model to the cascading failure simulation model. In particular, Section 2.1 describes the line failure mechanism and its stochastic representation. s 2.2 and 2.3 illustrate the cascade model and the restoration strategy, respectively, while the Sequential Monte Carlo procedure is presented in Section 2.4. The IEEE14 test network specifications and the results are described in Section 3. In Section 4, some conclusions are drawn.

\section{The model}

In this Section, we illustrate the framework for stochastic modeling of the cascading failure behavior of a transmission power network under uncertain weather conditions. In cascading failure analyses, the power transmission network is typically modeled as a graph $G=(V, E)$, where $V$ represents the set of vertices and $E$ the set of edges. Usually, it is assumed that only elements belonging to one of the sets $V$ or $E$ are subjected to failure and, correspondingly, the analyses are carried on under the assumptions of, respectively, node removals or edge removals (examples of these two different approaches can be found in [22-24] and [20,2527], respectively). In this work, we restrict our attention only to edge removals, since power transmission line failures are more common than bus failures [28].

The proposed framework aims at modeling the effects of random line failures due to both normal operation and extreme weather conditions in a power transmission network, accounting also for the subsequent restoration processes. The uncertainty in the occurrence of the failure events affects the network system reliability/availability in terms of the expected energy not served (EENS [GWh/y]), the average load shed due to failure events (ALS [MW/occ]), the average frequency of the power interruptions (AFF $[$ occ/y]) and their average duration (ART [h/y or h/occ]), which will be defined in Section 3.

The scheme of the conceptual model adopted is shown in Fig. 1. The deterministic cascading failure model allows the computation of the consequences of the failure of an individual line of a given network. The input to this model is the multivariate distribution of the sequence of initial line failure occurrences, $\boldsymbol{F}_{y}=\left\{f_{1}, f_{2}, \ldots, f_{n}\right\}$, where $n$ is the total number of line failure events over a given time horizon (assumed to be one year in this work), with each sequence element $f_{i}=\left\{t_{i}, l_{i}\right\}, i=1, \ldots, n$, repre- senting both the occurrence time $t_{i}$ and the network line affected by the failure $l_{i}, i=1, \ldots, N_{L}$, with $N_{L}$ being the total number of lines of the transmission network under consideration. The sequence of failure occurrences is determined, in turn, by the combination of a "normal" line failure model, representing, for example, failures due to aging, malfunctioning, human errors and attacks in general, and the "extreme weather" failure stochastic model, where the line failures are related to the random occurrence of extreme weather events, represented by the sequence $\boldsymbol{E}=\left\{e_{1}, e_{2}, \ldots, e_{m}\right\}$, where $m$ is the total number of events over the given time horizon (one year) and $e_{j}=\left\{t_{j}, p_{j}, I_{j}\right\}, j=1, \ldots, m$ is a sequence of elements representing the occurrence time $t_{j}$, the type of weather event $p_{j}$ and its intensity $I_{j}$. Similarly to [16] and [29], the severe weather events considered in this work are exceedingly high winds due to storms and lightning, under the assumption that the entire network experiences the same weather conditions.

An original deterministic restoration model is, then, coupled to the cascading failure model, so that, when a cascading failure extinguishes, the line initially failed is repaired and the network is assumed to re-start from "as good as new" conditions. As it will be shown in Section 2.3, the restoration process is also affected by the stochastic weather conditions.

Note that, since the input $\boldsymbol{F}_{y}$ is a stochastic, multivariate variable described by its probability density function (pdf) $d\left(\boldsymbol{F}_{y}\right)$, the outputs of the deterministic cascading failure model, i.e., the reliability/availability indicators EENS, ALS, ART and AFF, are also uncertain and described by pdfs d(EENS), d(ALS), $d($ ART) and $d(\mathrm{AFF})$, respectively.

\section{1. "Normal" and extreme-weather stochastic models of failure}

The random occurrence of "normal" failures is modeled as a homogeneous Poisson process, as proposed in [16]:

$P\left(N_{f}(t)=k\right)=\frac{\left[\lambda_{\text {norm }} * t\right]^{k}}{k !} e^{-\lambda_{\text {norm } * t}}, k=0,1,2 \ldots$

where $N_{f}(t)[$ occ $/ \mathrm{km}]$ is the number of failures per kilometer of grid line occurring in the time period $(0, \mathrm{t}], \lambda_{\text {norm }}[\mathrm{occ} / \mathrm{km} / \mathrm{h}]$, assumed to be constant throughout one year, is the failure rate under normal weather conditions per kilometer of grid line and $P\left(N_{f}(t)=k\right)$ is the probability that $k$ failures occur in the period $(0, t]$, per kilometer of grid line.

On the other hand, the random occurrence of extreme weather event-related failures [16] is modeled as a non-homogeneous Poisson process with a parameter $V_{e}(t)$ dependent on the stochastic weather conditions during the year. The weather conditions are determined by the stochastic sequence of weather events $\boldsymbol{E}_{y}=\left\{e_{1}, e_{2}, \ldots, e_{m}\right\}$. Similarly to [16], we model the occurrence of

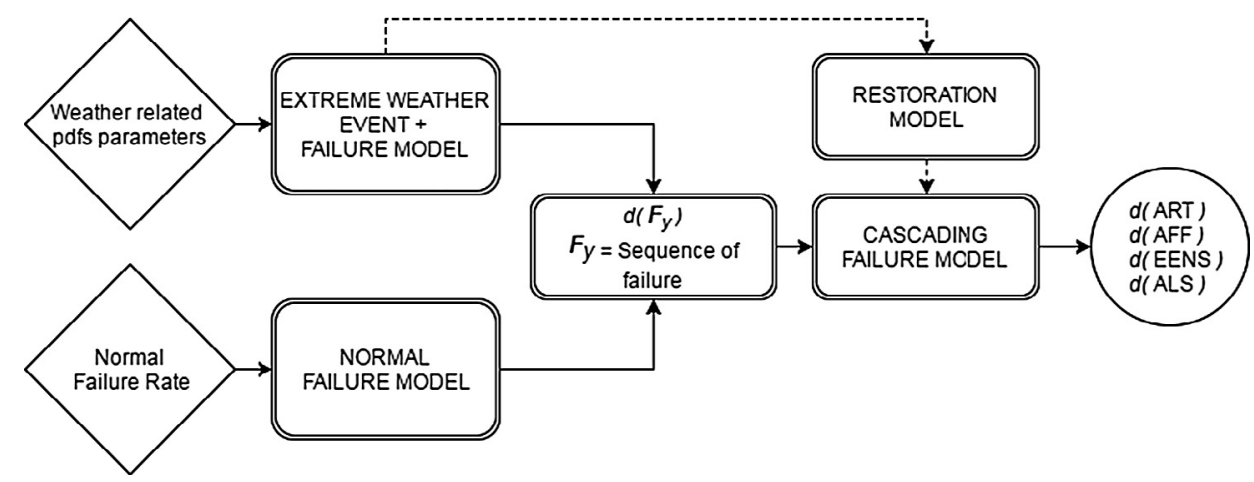

Fig. 1. Conceptual scheme of the proposed stochastic framework for the estimation of power grid reliability/availability indices. 
two types of events, i.e. exceedingly high winds and lightning, assuming they are independent. Wind storms are assumed to give rise to the falling of trees not properly trimmed and to damage structures like cables and poles, whereas lightning may directly damage the electrical equipment.

The occurrence of the weather events is modeled by a nonhomogeneous Poisson Process (NHPP) [1]:

$P\left(N_{p}(t)=k\right)=\frac{\left[V_{p}(t)\right]^{k}}{k !} e^{-V(t)}, k=0,1,2 \ldots$

where $N_{p}(t)$ [occ] is the number of events of type $p$ occurring in the time period $(0, \mathrm{t}], P\left(N_{p}(t)=k\right)$ is the probability that $k$ events of type $p$ occur in the period $(0, t]$ and $V_{p}(t)$ [occ] is the average number of occurrences of event $p$ over the time period $(0, t]$. The timedependent parameter $V_{p}(t)$ can be expressed as:

$V_{p}(t)=\int_{0}^{t} v_{p}\left(t^{*}\right) d t^{*}$

where $v_{p}(t)[\mathrm{occ} / \mathrm{h}]$ is the rate of occurrence of event $p$ (wind storms or lightning), taken to be stepwise constant on a monthly basis in order to account for the seasonality of the weather events. Fig. 2, taken from [16], shows the value of the rates of occurrence of wind storms and lightning as a function of time, used in this work. According to [16], the identification of the parameters used in the stochastic models described above is based on the weather observations collected in the Swedenergy [30] and the SMHI (i.e. the meteorological agency of Sweden) databases [31] and referring to two locations in the Swedish East and West coasts. Failure and weather statistics were gathered separately and, then, connected into a database, obtaining the weather conditions to which the grid was exposed when a line failed.

The occurrences of each type of weather event are, then, characterized by stochastic intensity levels and durations [16]. To represent the intensity level of a wind event, the maximum mean wind speed over a 10 -min period measured during a three-hour period is used. The lightning intensity is quantified through the number of cloud-to-ground flashes per kilometer and hour that affects the service areas.

According to [16], a wind storm event is considered to occur each time the wind speed (intensity) overcomes a critical threshold $w_{\text {crit }}$ : in fact, high winds give rise to failures only when the friction force acting on trees and poles overcome a critical value. Here, similarly to [16], we take $w_{\text {crit }}=8[\mathrm{~m} / \mathrm{s}]$, since it is observed that, above this value, the number of failures due to wind rapidly increases with wind speed. Thus, operatively, it is assumed that the random variable $w_{\text {wind }}[\mathrm{m} / \mathrm{s}]$, i.e. the wind intensity conditional on the occurrence of a wind storm event, can be written as:

$w_{\text {wind }}=w_{\text {crit }}+\Delta w$

where $w_{\text {crit }}$ is fixed and $\Delta w$ is the random variable assumed to be described by the Weibull distribution with the parameters reported in Table 1 . The durations of the wind storms $\left(D_{\text {wind }}\right)$ events are assumed to follow a Weibull distribution with the parameters reported in Table 1.

On the other hand, the lightning intensity level is quantified through the number of flashes that hit the ground per squarekilometer and hour $N_{g}(t)$ [occ $\left./ \mathrm{h} / \mathrm{km}^{2}\right]$, which is assumed to be a random variable distributed according to the Weibull distribution with the parameters reported in Table 1 [16]. As opposed to the high wind events, lightning gives rise to line failures regardless its ground flash density, so that no critical threshold is considered. The durations of the lightning events $\left(D_{\text {light }}\right)$ are assumed to follow a lognormal distribution with the parameters reported in Table 1.

The number of line failure occurrences due to each type of event is assumed to be distributed according to a NHPP:

$P\left(N_{f}(t)=k\right)=\frac{\left(\int_{0}^{t} \lambda_{f}\left(t^{*}\right) d t^{*}\right)^{k}}{k !} e^{-\int_{0}^{t} \lambda_{f}\left(t^{*}\right) d t^{*}}, k=0,1,2 \ldots$

where $N_{f}(t)$ is the number of line failures per kilometer occurring in the time period $(0, \mathrm{t}], \lambda_{f}(t)[\mathrm{occ} / \mathrm{km} / \mathrm{h}]$ is the failure rate per kilometer at time $t$ and $P\left(N_{f}(t)=k\right)$ is the probability that $k$ failures occur in the period $(0, t]$ per kilometer.

According to [16], since the failure processes are due to independent, different physical phenomena, then the total failure rate $\lambda(t)$ can be written as:

$\lambda(t)=\lambda_{\text {norm }}+\lambda_{\text {wind }}\left(w_{\text {wind }}(t)\right)+\lambda_{\text {light }}\left(N_{g}(t)\right)$

where $\lambda_{\text {wind }}\left(w_{\text {wind }}(t)\right)$ represents the line failure rate per kilometer due to wind storms at time $t$ and $\lambda_{\text {light }}\left(N_{g}(t)\right)$ is the line failure rate

Table 1

Parameters of the pdfs characterizing the extreme weather model [16].

\begin{tabular}{llll}
\hline & Distribution & Scale, $a$ & Shape, $b$ \\
\hline Wind storm duration $\left(D_{\text {wind }}\right)$ & Weibull & 9.89 & 1.17 \\
Wind storm intensity $(\Delta w)$ & Weibull & 1.23 & 1.05 \\
Lightning duration $\left(D_{\text {light }}\right)$ & Weibull & 0.96 & 0.85 \\
& & $\mu$ & $\sigma$ \\
Lightning intensity $\left(N_{g}(t)\right)$ & Lognormal & -5.34 & 1.07 \\
\hline
\end{tabular}

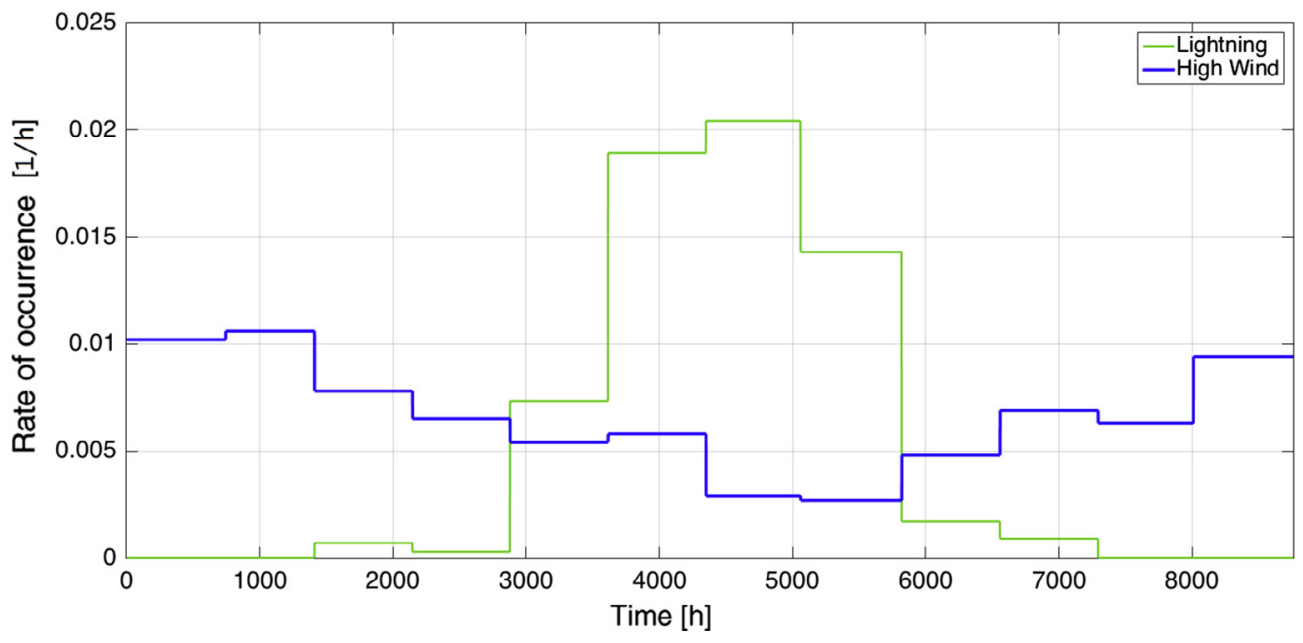

Fig. 2. Rates of occurrence for wind storms and lightning events estimated from weather data [16]. 
per kilometer due to lightning at time $t$. The failure rates $\lambda_{\text {wind }}$ and $\lambda_{\text {light }}$ depend on the occurrence of the weather events as follows.

A. Wind storm failure rate: The effects of wind speed are (i) increased pressure over trees, possibly falling on overhead lines and/or (ii) direct friction on poles and lines of the system, possibly making them fall or touch external bodies. According to [16] and to the previous work in [12], a model proportional to the square of wind velocity is used, resembling the equation for the drag force acting on an object in a fluid:

$\lambda_{\text {wind }}\left(w_{\text {wind }}(t)\right)=\alpha\left(\frac{w_{\text {wind }}(t)^{2}}{w_{\text {crit }}^{2}}-1\right) \lambda_{\text {norm }}$

where $\alpha$ is a positive scaling parameter. When either there are no wind events or the wind speed $w$ is below $w_{\text {crit }}$, then $\lambda_{\text {wind }}=0$.

B. Lightning failure rate: Lightning directly hits the subcomponents of the network, causing their failures. Similarly to [16] and to the previous work in [14], a linear relationship is used between the failure rate due to lightning $\lambda_{\text {light }}\left(N_{g}(t)\right)$ and the ground flash density $N_{g}(t)$ :

$\lambda_{\text {light }}\left(N_{g}(t)\right)=\left(\beta N_{g}(t)\right) \lambda_{\text {norm }}$

where $\beta$ is a positive scaling parameter. When there are no lightning events, then $\lambda_{\text {light }}=0$.

Note that a rare, but possible, event is the simultaneous occurrence of a light storm and a high wind event. According to (6) the total failure rate $\lambda(t)$ is given by the summation of all the three contributions.

The specific failure rate of the generic $i$-th line of length $l_{i}, i=1, \ldots, N_{L}$ is, then:

$\lambda_{i}(t)=\lambda(t) * l_{i}$

\subsection{Cascading failure model}

In this paper, we resort to the cascading failure model developed in [33] and applied also in [32]. The model simulates the evolution of cascading failures, triggered by an initial set of disconnected lines $L_{\text {disc }}$, relying on the direct current (DC) power flow approximation [20]. Operatively, the disconnected set $L_{\text {disc }}$ includes both the line damaged by the failure $f_{i}$ and, possibly, other unavailable lines at time $t_{i}$ due to restoration. Starting from the initial grid $G^{0}=G$, the general cascade algorithm reads [33]:

1. $G^{1}=\left(V, M-\left\{L_{d i s c}\right\}\right)$. Compute vector $\boldsymbol{P}^{1}$ of power flow in $G^{1}$.

2. If required, adjust load and generation (re-dispatch).

3. Stage $r$. Compute $\boldsymbol{P}^{r}$ power flow vector in $G^{r}$.

4. Set $O^{r}$, i.e., the set of lines overloaded (and disconnected) at stage $r$.

5. If $\left|O^{r}\right| \geqslant 1$, set $G^{r+1}=\left(V, M^{r}-O^{r}\right)$ and go to step 2, otherwise, end the cascade.

The stage $r$ corresponds to a line disconnection event in the cascading failure simulation, which can result in lines disconnection $\left|O^{r}\right| \geqslant 1$ or not $\left|O^{r}\right|=0$. A re-dispatch action (step 3 ) is a consequence of the islanding process, which occurs when an entire portion of the network $G$ becomes disconnected from the grid, thus experiencing an excess either in the power supply or in the power demand. The vector $\boldsymbol{P}^{r}$ is computed throughout the cascade by means of the DC power flow model, a linear approximation of the AC power flow model, which relies on the following assumptions: $(i)$ all bus voltage phasors are assumed
1.0 per unit in magnitude, (ii) transmission line resistance is neglected and voltage angles differences are assumed to be small such that [34]:

$\forall k, j \in V \sin \left(\theta_{k}-\theta_{j}\right) \approx \theta_{k}-\theta_{j}$

where $\theta_{k}$ is the voltage angle at bus $k$. Let $\theta$ be the $n$-dimensional vector of voltage angles, where a component is chosen to be equal to 0 (reference bus), and $b_{k j}$ is the susceptance of the line joining node $k$ to node $j$. The $n \times n$ matrix $\boldsymbol{B}^{r}$ at the $r$-th iteration is defined by:

$$
\begin{gathered}
B_{k k}^{r}=\sum_{\text {bus j connected to bus } i} b_{k j}^{r} \\
B_{k j}^{r}=-b_{k j}
\end{gathered}
$$

The DC load flow equations are:

$$
\boldsymbol{W}^{r}=\boldsymbol{B}^{r} \boldsymbol{\theta}^{r}
$$

where $\boldsymbol{W}^{r}$ is the vector of the node power outputs, which sum to zero due to the power balance constraint between power generation and demand within the network. The matrix $\boldsymbol{B}^{r}$ has rank $N-1$; removing the row corresponding to the reference bus and taking the inverse, one obtains:

$\boldsymbol{\theta}^{r}=\boldsymbol{X}^{r} \boldsymbol{W}^{r}$

Finally, the power flow in line $l_{i}$ connecting bus $k$ and $j$ is computed as:

$P_{l_{i}}^{r}=b_{k j}^{r}\left(\theta_{k}^{r}-\theta_{j}^{r}\right)$

For further details on the DC power flow approximation, the interested reader may refer to [34].

During the propagation of the cascading failure, the grid could become separated in multiple connected components, also called islands [33]. The newly formed islands may not satisfy the power balance, thus not allowing further DC computation. A simple redispatch routine is embedded in the model to overcome this issue, as follows. Considering the island $I \subset G$, where $\left(\operatorname{Gen}_{I}, \operatorname{Load}_{I}\right)$ are the sets of generator and load buses in $I$, at each iteration $r$ three different unbalanced situations could occur:

1. The power supply does not meet the demand but the generators have enough reserve to accommodate the surplus:

$\sum_{k \in G e n_{I}} P G_{k}^{r-1}<\sum_{k \in \text { Load }_{I}} P D_{k}^{0}$ and $\sum_{k \in \text { Load }_{I}} P D_{k}^{0}<\sum_{k \in G e n_{I}} P M A X_{k}$

Then, the power production of each generator belonging to $I$ is increased proportionally:

$$
\begin{aligned}
& \forall k \in \text { Gen }_{I}: P G_{k}^{r}=P G_{k}^{r-1} \\
& \quad+\frac{P M A X_{k}-P G_{k}^{r-1}}{\sum_{k \in G e n_{I}} P M A X_{k}-\sum_{k \in G e n_{I}} P G_{k}^{r-1}}\left(\sum_{\text {Load }_{I}} P D_{k}^{0}-\sum_{\text {Load }_{I}} P G_{k}^{r-1}\right)
\end{aligned}
$$

$\forall j \in \operatorname{Load}_{I}: P D_{j}^{r}=P D_{j}^{0}$

2. The power supply does not meet the demand and does not have enough reserve to meet the surplus:

$\sum_{k \in G e n_{I}} P G_{k}^{r-1}<\sum_{k \in \text { Load }_{I}} P D_{k}^{0}$ and $\sum_{k \in \text { Load }_{I}} P D_{k}^{0}>\sum_{k \in \text { Gen }_{I}} P M A X_{k}$

Then, the power demand of each load belonging to $I$ is decreased proportionally and the power supply is fixed to the maximum limit: 
$\forall k \in \operatorname{Load}_{I}: P D_{k}^{r}=P D_{k}^{0} \frac{\sum_{k \in \operatorname{Gen}_{I}} P M A X_{k}}{\sum_{k \in \text { oad }_{I}} P D_{k}^{0}}$

$\forall j \in$ Gen $_{I}: P G_{j}^{r}=P M A X_{j}$

Meanwhile, if in the island $I$ the power supply exceeds the demand, i.e.:

$\sum_{k \in G_{I} n_{I}} P G_{k}^{r-1}>\sum_{k \in \text { Load }_{I}} P D_{k}^{0}$

then, the power production of each generator belonging to $I$ is decreased proportionally:

$\forall k \in \operatorname{Gen}_{I}: P G_{k}^{r}=P G_{k}^{r-1} \frac{\sum_{k \in \text { Load }_{l}} P D_{k}^{0}}{\sum_{k \in G_{\text {Gen }}} P G_{k}^{r-1}}$

$\forall j \in \operatorname{Load}_{I}: P D_{j}^{r}=P D_{j}^{0}$

In all the above equations, $P D_{j}^{0}$ denotes the power demand of node $j$ before the initial failure.

The major drawback of this strategy is related to the fact that it does not take into account the capacities of the surviving lines in the system, in order to more effectively re-dispatch the power flows. For example, in [20] the power supply and demand is adjusted by means of a linear optimization, whereas in [35] a controller proportionally sheds load or increases generation depending on the situation. However, with this strategy, the simulation of cascading failures are much faster, thus allowing to demonstrate the feasibility of the statistical analyses and the optimizations presented in this paper at reasonable computational efforts.

At step 4 of the procedure for simulating cascading failures, an outage model is required to identify the transmission lines which fail during iteration $r$. To this aim, we exploit the concept of effective power flow $[33,36]$, where the transients of the power flows $x_{l_{i}}$ in the network lines after a disconnection event are modeled as:

$x_{l_{i}}(t+1)=(1-\alpha) x_{l_{i}}(t)+\alpha P_{l_{i}}^{r} ; l \in E^{r}$

$x_{l_{i}}(0)=H_{l_{i}}^{r-1}$

where the parameter $\alpha \in(0,1]$ is called thermal inertia of the grid, $P_{l_{i}}^{r}$ is the $i$-th component of the power flow vector $\boldsymbol{P}^{r}, t$ is a discrete time index and $H_{l_{i}}^{r-1}$ is the power flowing through line $l_{i}$ at the $r-1$ -th failure event. The grid inertia $\alpha$ is a parameter introduced in order to model the "reactiveness" or "memory" of the grid with respect to any changes: if $\alpha=1$, the system is memoryless and $x_{l_{i}}(t+1)=P_{l_{i}}^{r}$, thus implying an instantaneous power flow change after the $r$-th failure event, whereas $\alpha<1$ implies a transient phase which becomes longer as $\alpha$ approaches 0 [33]. If $x_{l_{i}}(t)$ is larger than line capacity, then line $l_{i}$ fails and the propagation of the failure cascade continues. If multiple lines are overloaded, the line undergoing the fastest transient is disconnected; otherwise, if no line is overloaded, the cascade stops.

In this paper, for simplicity, the cascade is assumed instantaneous, i.e. its duration can be neglected with respect to the restoration times (see next Section).

\subsection{Restoration model}

Each time the network suffers a damage, the initially failed line is repaired, so that the network is restored to its initial conditions. We assume that: $(i)$ to perform the reconnection, a crew of repairmen is dispatched as soon as the cascade ends; (ii) the network becomes fully functional as soon as the initial line is repaired; (iii) the duration of the cascade can be neglected with respect to the times of repairs.

The variability of the weather conditions introduces uncertainties in the time required for the repair, as adverse environmental conditions usually slow down or even prevent the crew from intervening. The restoration time is the overall time needed to reactivate the line, i.e. the total time required for the arrival of the crew, for the repair itself, and for switching back and reactivating all the disconnected components until the initial steady state is reached again. In case of multiple failures or sequential attacks to the networks, it is assumed that multiple crews can be dispatched to repair the failed lines.

The dependence of the repair times on the weather conditions is modeled by means of a restoration velocity $v_{\text {rest }}$, defined as the "portion" of the total required repair effort carried on in one hour. For simplicity, but with no loss of generality, we assume that the failed lines always require the same amount of repair effort to be fully restored, regardless their type or length. The restoration velocity, on the other hand, depends on the severity of the natural disturbances under which the repair is carried on. Under normal circumstances, the restoration velocity for overhead-uninsulated lines is assumed to be fixed, and equal to $v_{\text {norm }}=20[\% / \mathrm{h}]$ or, in other words, the undisturbed repair time is always $t_{\text {rep,norm }}=5$ [h], in line with what suggested in [37]. Given a 1-year sequence of weather events and line failures, if the repairs take place during a severe weather occurrence, then, a new, lower restoration velocity $v_{\text {rest }}$ is assumed for the repair of the failed line:

$$
v_{\text {rest }}(t)= \begin{cases}\frac{v_{\text {norm }}}{1+\varepsilon\left(w_{\text {wind }}(t)-w_{\text {crit }}\right)}, & \text { for } w(t)>w_{\text {crit }} \text { and } N_{g}=0 \\ \frac{v_{\text {norm }}}{1+\varphi N_{g}}, & \text { for } w(t)\left\langle w_{\text {crit }} \text { and } N_{g}\right\rangle 0 \\ \frac{v_{\text {norm }}}{\left[1+\varepsilon\left(w_{\text {wind }}(t)-w_{\text {crit }}\right)\right]+\left[1+\varphi N_{g}\right]}, & \text { for } w(t)>w_{\text {crit }} \text { and } N_{g}>0\end{cases}
$$

where $\varepsilon$ and $\varphi$ are parameters with positive values. As the intensity of the severe weather events grows, the restoration velocity decreases, increasing the duration of the outage and the interruption in the power delivery. During a given realization of a sequence of weather events and failures, when the weather conditions go back to normality, the restoration velocity is again set to $v_{\text {norm }}$. For example, if a line failure occurs during a high wind event and the repair is not finished before the weather event ends, then the repair action continues with the normal restoration velocity $v_{\text {norm }}$; on the contrary, if an outage occurs under normal circumstances and a natural disturbance takes place before the restoration is completed, the restoration of the line continues with a lower velocity.

In the proposed model, we further assume that, while a line of the grid is under repair, the other components of the network are still subjected to failure, in agreement with the "failure bunching due to adverse weather" concept illustrated in [10]. In case this happens, the network is in a weaker configuration and the failure of another component has, in general, even more severe consequences, also depending on whether it affects the disconnected portion of the grid or the still operating part. In the first case, no further damages are considered, since no power is dispatched and no further load can be shed. In the second case, the failure of another line and the possible, consequent cascade likely lead to more catastrophic consequences, as a smaller subset of lines is available for the re-dispatching of the power supply. The repairs of all the failed lines are assumed to be simultaneously carried on at the same velocities. In conclusion, the occurrence of severe weather events causes, on average, the restorations to last longer, due to the combined effects of a reduced $v_{\text {rest }}$ and the possible occurrence of multiple failures.

For the following case study of Section 3 below, the parameters defining the restoration velocity in disturbed conditions (28) are 
arbitrary set to $\varepsilon=0.4$ and $\varphi=40$, on the basis of engineering common sense.

\subsection{Sequential time Monte Carlo}

The complete model, i.e., the stochastic model of line failures driven by "normal" and extreme weather events combined with the DC power flow-based cascading failure model, lends itself to a simulation-based numerical resolution scheme for estimating the expected values of the reliability/availability indices of interest. To this aim, similarly to [16] and [17], we propose to resort to a customized sequential time Monte Carlo scheme for generating repeated realizations of several histories of the life of a power transmission network. In what follows, we briefly describe the solution method, whose flowchart is shown in Fig. 3. For further details on the procedure, the interested reader may refer to [16] and [17].

The sequential Monte Carlo scheme proceeds as follows. The time horizon of the simulation is 1 year, i.e., $8760 \mathrm{~h}$. At the beginning of each year, the grid is assumed to be fully operational and the weather conditions to be normal. First, the times of occurrences of the "normal" failures throughout the year are generated

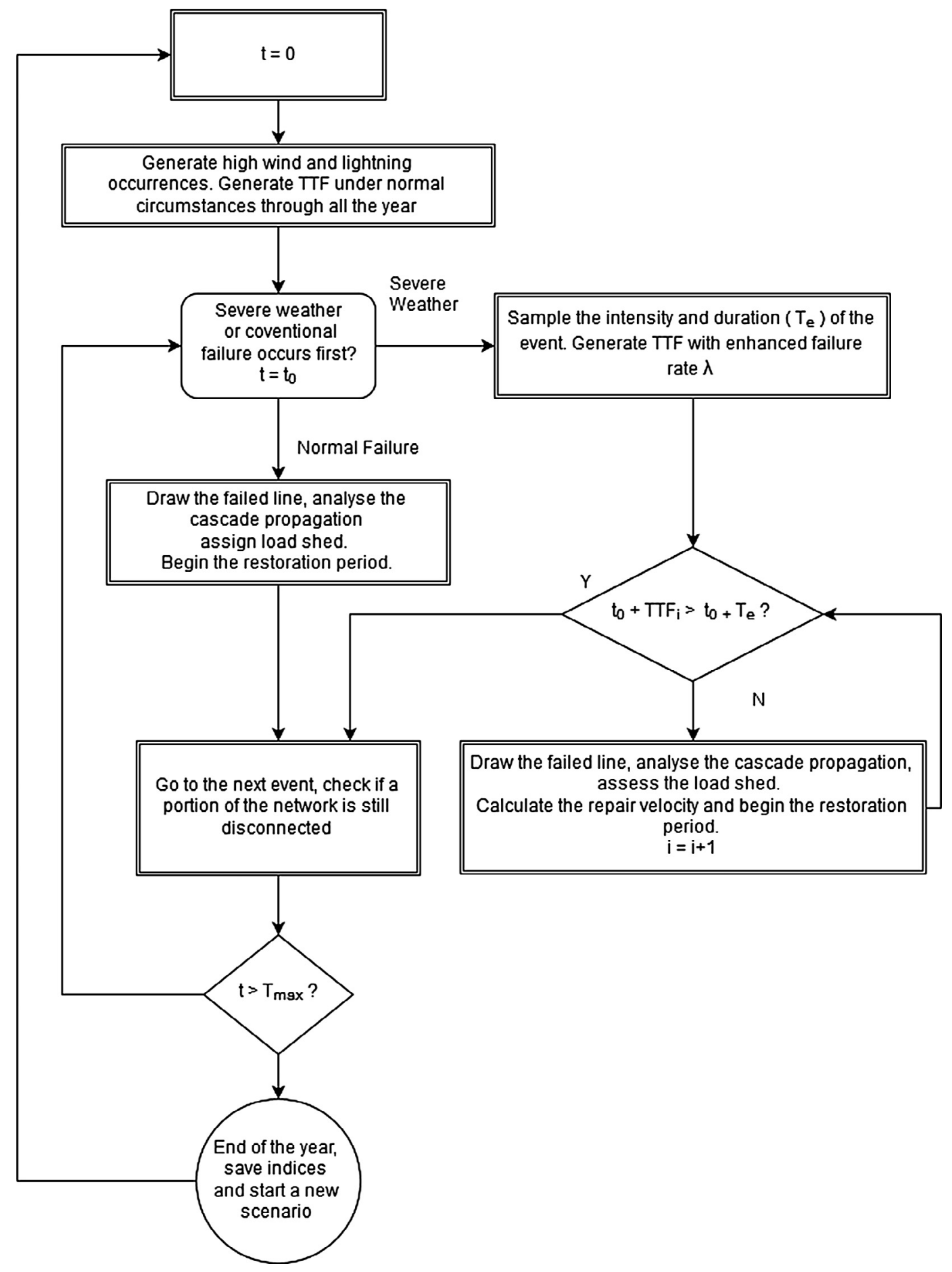

Fig. 3. Flowchart of the Monte Carlo simulation algorithm. 
by sampling from the exponential distribution associated to the homogeneous Poisson distribution (1) with total parameter $\lambda_{\text {norm }}$ Tот $=\sum_{i}^{N_{L}}\left(\lambda_{\text {norm }_{i}}\right)$, where $\lambda_{\text {norm }_{i}}$ is the failure rate of line $i$ due to "normal" causes. The failed line $i$ of length $l_{i}$ is sampled from a

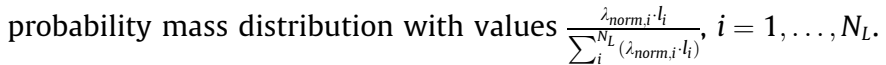
Then, the occurrence times of the two types of severe weather events are independently sampled from the distributions associated to the non-homogeneous Poisson distributions (2) with parameters $v_{\text {wind }}(t)$ and $v_{\text {light }}(t)$, respectively. The intensities and durations of the weather events in the annual sequence are sampled from the corresponding distributions (Table 1). The times of the failures possibly occurring during each weather event are sampled from the Poisson distribution with total parameter $\lambda_{p_{\text {TOT }}}=\sum_{i}^{N_{L}}\left(\lambda_{p}(t)_{i}\right)$, where $p$ refers to the extreme weather event type. If the sampled failure time falls within the duration of the extreme weather event under consideration, then, the sample is accepted; otherwise, it is rejected. The line of the grid actually undergoing the failure is, then, sampled from the probability mass distribution built similarly to the case of the "normal" failures described above.

Finally, the three sequences of events are superimposed. In correspondence of each failure of the sequence, the cascading failure model is run until a new, stable configuration is found. At the end of the cascading failure event, the repair of the initial line starts with a restoration velocity $v_{\text {rest }}(t)$ computed according to the model described above (28). The possible occurrence of multiple failures within the same weather event is treated as described at the end of Section 2.3.

\section{Application}

The proposed modeling and simulation framework is applied to the IEEE14 test case [21]. The grid model is composed by two kinds of components, nodes and lines, which are representative of all the subcomponents. The test network represents a power transmission system with 20 links $\left(N_{L}=20\right)$, mainly overhead, uninsulated lines. The system operates with two different voltage levels, 132 and $230 \mathrm{kV}$ : to allow this, transformers are present. Each node represents a generating unit, a load point or a transmission/switch bus. The specific topology and data regarding generating capacity can be found in [21]. In the following, we shall use $G$ to denote the topology $\left(V, N_{L}\right)$ of the power network, with $|V|=14$ nodes and $N_{L}$ links, and its complete configuration, i.e. the network topology, loads and generators. Although the size of the system is small, its composition is regarded complete with respect to the characteristics of interest for the purpose of the modeling and simulation framework development proposed in the present work. In order to compute the failure probabilities, the length of the lines is needed. It is possible to infer the line length from the available data in [21], using the total impedance $Z$ reported in both [38] and [39]:

$Z_{i}=\left(r_{i}+j x_{i}\right) l_{i}$

where $Z_{i}$ is the impedance of the line $i, r$ and $x$ are the resistance and reactance per unit of length, respectively. Assuming as power base $S_{\text {base }}=100$ [MVA] [21] and using the nominal voltage $V_{\text {base }}$ of each line, the base impedance $Z_{\text {base }}$ can be obtained:

$Z_{\text {base }}=\frac{V_{\text {base }}^{2}}{S_{\text {base }}}$

Multiplying $Z_{\text {base }}$ for the specific reactance of each line and using a conversion factor of $0.7[\Omega /$ mile], the length of each line can be inferred, as proposed in [40] and already applied to the IEEE14 network in [39]. Lines containing transformers are considered to have null length.

Table 3 shows the main network data, i.e. the lines types, their operating voltages and lengths, the corresponding "normal" failure rates $\lambda_{\text {norm }}$ (assumed to be related only to the line voltage level, as proposed in [41] and also used in [39]) and the resulting annual failure probabilities. The failure rates parameters in (7) and (8) are chosen to be $\alpha=1.13 \cdot 10^{2}$ and $\beta=3.10 \cdot 10^{3}$, on the basis of engineering common sense. A total of $N_{S}=4000$ histories of one year life of the IEEE14 network are simulated, with the proposed Monte Carlo simulation scheme.

Fig. 4 shows the outcome of a realization of weather events and line failures occurrences during a portion of one year. Each squared wave represents the occurrence of an event, its starting time and duration. The solid line is referred to the occurrence of a wind storm, the circles indicate the weather-related failure times and the dashed line is associated to the consequent restoration processes. In this particular realization, the wind storm event gives rise to multiple line failures. The event is characterized by a wind speed of $w_{\text {wind }}=9.42[\mathrm{~m} / \mathrm{s}]$, lasting for $D_{\text {wind }}=31.6[\mathrm{~h}]$ and causing four lines failures. The corresponding restoration velocity, computed by $(28)$, is $v_{\text {rest }}(t)=0.638$, which would imply a repair duration $t_{\text {rep }}(t)=7.84[\mathrm{~h}]$. However, after the occurrence of the initial

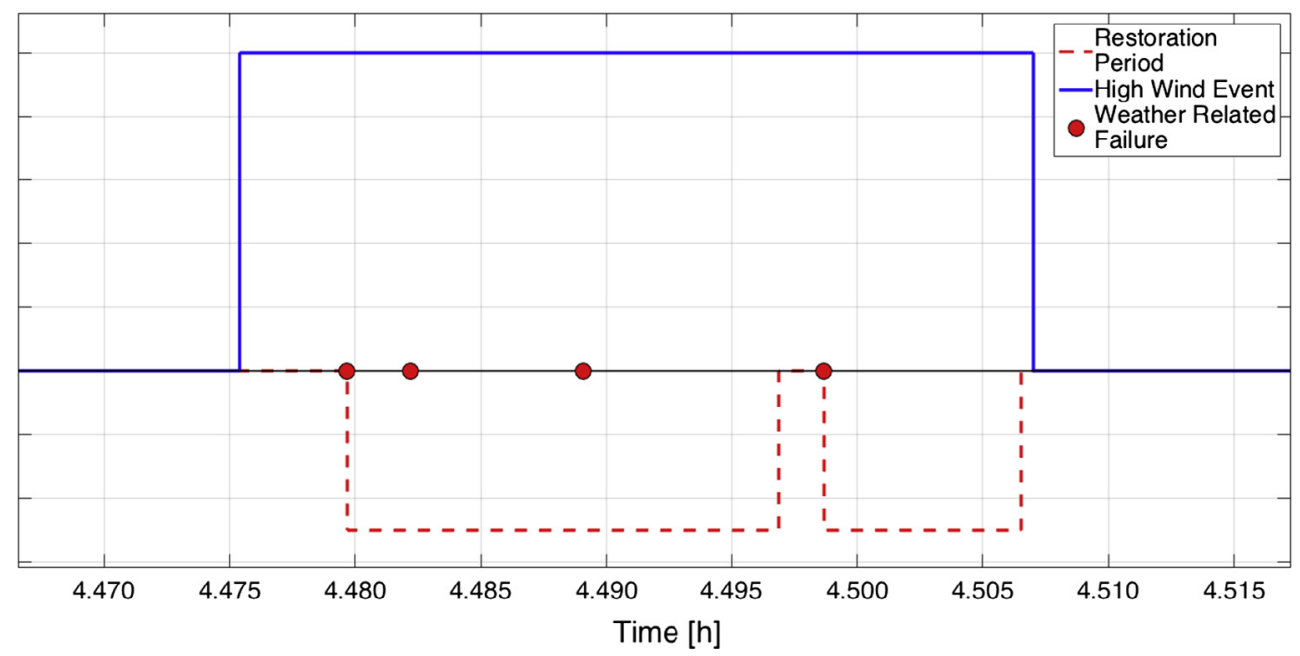

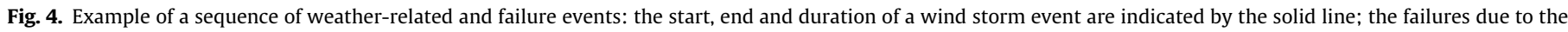
wind storms are represented by the circles; the start, the end and the duration of the repairs are indicated by the dashed line. 
Table 2

Details of the sequence of Fig. 4.

\begin{tabular}{|c|c|c|c|c|}
\hline Failure & Time $[h]$ & Line Targeted $l_{i}$ (unavailable lines) & Cascade lines $l_{i}$ disconnection & Load shed $[M W$ \\
\hline 1 & 4479.7 & 12 & - & 0 \\
\hline 2 & 4480.2 & $3(12)$ & 6 & 9.54 \\
\hline 3 & 4489.0 & $13(3,6,12)$ & $19,18,17,16$ & 136.47 \\
\hline 4 & 4498.7 & 17 & 20 & 16.11 \\
\hline
\end{tabular}

Table 3

Data for the IEEE14 transmission network ([39] and [41]).

\begin{tabular}{|c|c|c|c|c|c|}
\hline Node $i$ & Node $j$ & Line type and voltage & Length $[\mathrm{km}]$ & Failure rate $\lambda_{\text {norm }}[1 / \mathrm{km} / \mathrm{y}]$ & Annual failure probability ("normal") \\
\hline 1 & 2 & $132 \mathrm{kV}$ line & 22 & $1.086 \cdot 10^{-2}$ & 0.216 \\
\hline 1 & 5 & $132 \mathrm{kV}$ line & 81 & $1.086 \cdot 10^{-2}$ & 0.585 \\
\hline 2 & 3 & $132 \mathrm{kV}$ line & 72 & $1.086 \cdot 10^{-2}$ & 0.542 \\
\hline 2 & 4 & $132 \mathrm{kV}$ line & 64 & $1.086 \cdot 10^{-2}$ & 0.501 \\
\hline 2 & 5 & $132 \mathrm{kV}$ line & 63 & $1.086 \cdot 10^{-2}$ & 0.495 \\
\hline 3 & 4 & $132 \mathrm{kV}$ line & 62 & $1.086 \cdot 10^{-2}$ & 0.490 \\
\hline 4 & 5 & $132 \mathrm{kV}$ line & 15 & $1.086 \cdot 10^{-2}$ & 0.150 \\
\hline 4 & 7 & $132 / 230 \mathrm{kV}$ transformer & - & - & - \\
\hline 4 & 9 & $132 / 230 \mathrm{kV}$ transformer & - & - & - \\
\hline 5 & 6 & $132 / 230 \mathrm{kV}$ transformer & - & - & - \\
\hline 6 & 11 & $230 \mathrm{kV}$ line & 219 & $5.429 \cdot 10^{-2}$ & 0.696 \\
\hline 6 & 12 & $230 \mathrm{kV}$ line & 282 & $5.429 \cdot 10^{-2}$ & 0.784 \\
\hline 6 & 13 & $230 \mathrm{kV}$ line & 144 & $5.429 \cdot 10^{-2}$ & 0.542 \\
\hline 7 & 8 & $132 / 230 \mathrm{kV}$ transformer & - & - & - \\
\hline 7 & 9 & $132 / 230 \mathrm{kV}$ transformer & - & - & - \\
\hline 9 & 10 & $230 \mathrm{kV}$ line & 93 & $5.429 \cdot 10^{-2}$ & 0.396 \\
\hline 9 & 14 & $230 \mathrm{kV}$ line & 298 & $5.429 \cdot 10^{-2}$ & 0.802 \\
\hline 10 & 11 & $230 \mathrm{kV}$ line & 212 & $5.429 \cdot 10^{-2}$ & 0.684 \\
\hline 12 & 13 & $230 \mathrm{kV}$ line & 220 & $5.429 \cdot 10^{-2}$ & 0.697 \\
\hline 13 & 14 & $230 \mathrm{kV}$ line & 384 & $5.429 \cdot 10^{-2}$ & 0.876 \\
\hline
\end{tabular}

failure at $t=4479.7$ [h], a second and third failures occur before the conclusion of the repair of the first failure event. Since the reconnection of all the disconnected lines is assumed to be performed at the same time (Section 2.3), any further line failure, occurring within the restoration period of a previous damage, prevents the repairs to be finished on schedule and a new restoration period begins. In the presented case, the three successive failure events result in a restoration duration of $t_{\text {rep }}(t)=17.2$ [h]. Table 2 reports the storyline corresponding to the failure sequence shown in Fig. 4, giving the failures occurrence times (column 1), the grid line damaged by the severe weather event (column 2), the lines disconnected after the cascading failure events (column 3 ) and the load shed after each cascading failure. It can be seen that the size of the subset of disconnected lines increases from the first to the third failure and, accordingly, also the undelivered power does. The fourth and last failure, instead, occurs after the repairs are completed, so that a larger subset of lines is again available for re-dispatching the power and, consequently, the final power shed is lower.

As anticipated in Section 2, we quantify the reliability/availability of the power transmission network by means of the following indices: ( $i$ ) the System Average Restoration Time Index (ART), (ii) the System Average Failure Frequency Index (AFF) and (iii) the Expected Energy Not Supplied (EENS).

The ART indicator is estimated in two different versions: $A_{\mathrm{RT}}$ $[\mathrm{h} / \mathrm{y}]$ and $\mathrm{ART}_{i}[\mathrm{~h} / \mathrm{occ}]$. The index $\mathrm{ART}_{y}[\mathrm{~h} / \mathrm{y}]$ is the average duration of the outages over one year:

$\widehat{A R T}_{y}=\sum_{j=1}^{N_{S}} \frac{\sum_{i}^{N_{e_{j}}} t_{r e p, e_{i}}}{N_{S}}$

where $N_{e_{j}}$ is the number of failure occurrences due to the event $e$ ("normal" or weather-related) in the year $j$ and $t_{r e p, e_{i}}$ is the restora- tion time associated to the cascade failure event $i$ caused by the event $e$. Note that the index $\mathrm{ART}_{y}$ bears some similarities with the SAIDI indicator typically used for quantifying the reliability of power distribution networks (see, for example, [16] and [17]). The index $\mathrm{ART}_{i}[\mathrm{~h} / \mathrm{occ}]$ is the average duration of a single outage, here estimated as:

$\widehat{\mathrm{ART}}_{i}=\frac{\sum_{i}^{N_{e, t o t}} t_{r e p, e_{i}}}{N_{e, t o t}}$

where $N_{e, t o t}$ is defined as the overall number of failure occurrences due to the event of type $e$ in all the $N_{s}$ simulations:

$N_{e, t o t}=\sum_{j=1}^{N_{S}} N_{e_{j}}$

The AFF [occ/y] index quantifies the average number of failure occurrences in the grid in one year, here estimated as:

$\widehat{A F F}=\frac{\sum_{i=1}^{N_{S}} N_{e_{i}}}{N_{S}}$

The EENS [GWh/y] indicator represents the annual average amount of energy not delivered by the transmission network due to the disruptive events, here estimated as:

$\widehat{E E N S}=\sum_{j=1}^{N_{S}} \frac{\sum_{i}^{N_{e_{j}}} L S_{e_{i}} * t_{r e p, e_{i}}}{N_{S}}$

Finally, since we are interested also in quantifying the average impact of cascading failures on the network reliability/availability, we further introduce the Average Load Shed index (ALS [MW/occ]) as the average power not served at the end of a failure and, possibly, subsequent cascade event, here estimated as: 
$\widehat{A L S}=\frac{\sum_{i=1}^{N_{e, t o t}} L S_{e_{i}}}{N_{e, t o t}}$

where $\mathrm{LS}_{e_{i}}$ [MW/occ] is the load shedding after a cascade event caused by the event $e$.

Note that the EENS indicator is widely used in analyses of both transmission and distribution grids and the ALS is a common measure of the impact of cascading failures in transmission grids.

Following the approach proposed in [16], in order to measure the contribution to the reliability/availability indices due to the extreme weather events, the simulations are carried on in two different settings: in the first case (case 1), we assume that the network is subjected only to "normal" failures, i.e. the cascades are triggered by line failures only due to normal operating conditions; in the second case (case 2), the occurrences of wind storms and lightning are included in the simulation.

With regards to case 2 , the estimates of the mean duration, $\widehat{D}_{e}$, and of the average number of occurrences of event $e, \hat{n}_{e}$, are computed as:

$\hat{n}_{e}=\frac{\sum_{i=1}^{N_{S}} n_{e, i}}{N_{S}}$

$\widehat{D}_{e}=\frac{\sum_{i=1}^{n_{e, t o t}} D_{e, i}}{n_{e, t o t}}$

where $n_{e, t o t}$ is the total number of events $e$ among all the $N_{S}$ simulations. The resulting average occurrences of high wind and lightning events are estimated to be $\hat{n}_{\text {wind }}=53.2$ [occ/y] and $\widehat{N}_{\text {light }}=48.5$ [occ/y], respectively, while their mean durations are $\widehat{D}_{\text {wind }}=9.78[\mathrm{~h}]$ and $\widehat{D}_{\text {light }}=1.21[\mathrm{~h}]$, respectively. The portion of the year interested by extreme weather events is:

$\widehat{T}_{\text {wind }}=\widehat{D}_{\text {wind }} * \hat{n}_{\text {wind }}=520[\mathrm{~h}]$

$\widehat{T}_{\text {light }}=\widehat{D}_{\text {light }} * \hat{n}_{\text {light }}=58[\mathrm{~h}]$

$\frac{\widehat{T}_{\text {wind }}+\widehat{T}_{\text {light }}}{8760 h}=6.6 \%$

Table 4 shows the results obtained in the two cases, where the symbol $\mu$ indicates the sample means of the reliability/availability indices and $\sigma$ their sample standard deviations.

Table 5 shows the individual contributions to the reliability/ availability indices due to the occurrence of each type of extreme weather event considered, including their possible simultaneous occurrence. In other words, the Table shows the values of the indicators computed by taking into account only the contribution of a single weather event typology, i.e., either only high winds, light- ning or their simultaneous occurrence (lightning during high winds).

The average number of failures in one year ( $\widehat{\mathrm{AFF}}$, column 3 , Table 4) is larger in case 2 , demonstrating that the extreme weather events have a significant impact on the reliability/availability performances of the network, even if their total average duration represents only a small fraction of one year, as shown above.

Other significant consequences of the severe weather events are the increases in the average durations of the single interruption of supply $\left(\widehat{\operatorname{ART}}_{i}\right)$ and in the annual average duration of the restoration processes $\left(\widehat{\mathrm{ART}}_{y}\right)$. It can be seen that the relative increase in $\widehat{\mathrm{ART}}_{i}$ is significantly smaller than that in $\widehat{\mathrm{ART}}_{y}$, being due only to the effects of the restoration model. In particular, the increase is due to the simultaneous effects of $(i)$ the lower restoration velocity $v_{\text {rest }}(t)$ during the weather event, directly correlated to its intensity and (ii) the occurrence of multiple line failures within the same extreme weather event, since more lines have to be repaired in order for the grid to be restored. On the other hand, the $\widehat{A R T}_{y}$ is also affected by the average annual number of interruptions.

As said above, each time the system is subjected to multiple failures within the same extreme weather event, it also experiences larger load shedding magnitudes at the end of the cascades, due to the progressive reduction of the available lines, as shown earlier. This is shown by the estimates of the load shed index $\widehat{\mathrm{ALS}}$, as shown in column 4 , Table 5 , where the separate contributions due to the different weather event types are reported. In fact, as the failure rate increases, the probability of multiple failures grows and the magnitude of the final outages increases. For example, the maximum value of the failure rate occurs when a wind storm and a lightning event occur simultaneously, and, coherently the maximum ALS is observed in correspondence of this kind of event. Note also that the duration of the weather event has a significant effect on the value of the index ALS. Comparing the $\widehat{A L S}$ index due to high wind, lightning (column 4, Table 5) and the one calculated during "normal" conditions (column 4, Table 4) it can be noticed that a sustained extreme weather event (as wind storms) can imply severe consequences, while a short one (such as lightning) causes an average load shed similar to the one under normal circumstances.

Another important consequence of the larger number of failures during extreme weather and of the associated larger restoration times, is a larger value of the index EENS, as it can be seen from Table 4 . Table 5 also shows that the wind storm events provide the largest contribution to EENS, mostly due to their longer durations, which significantly affect the related restoration velocities $\left(\widehat{D}_{\text {wind }}=9.36[\mathrm{~h}]\right)$.

Table 4

Results under normal failures. Average of 4000 repetitions of the simulations.

\begin{tabular}{|c|c|c|c|c|c|c|c|c|c|c|}
\hline \multirow[t]{2}{*}{ IEEE14 } & \multicolumn{2}{|c|}{$\widehat{\mathrm{ART}}_{i}[\mathrm{~h} / \mathrm{occ}]$} & \multicolumn{2}{|c|}{$\widehat{\operatorname{ART}}_{y}[\mathrm{~h} / \mathrm{y}]$} & \multicolumn{2}{|c|}{$\widehat{\mathrm{AFF}}[\mathrm{occ} / \mathrm{y}]$} & \multicolumn{2}{|c|}{$\widehat{\mathrm{ALS}}[\mathrm{MW} / \mathrm{occ}]$} & \multicolumn{2}{|c|}{$\widehat{\mathrm{EENS}}[\mathrm{GW} \mathrm{h}]$} \\
\hline & $\mu$ & $\sigma$ & $\mu$ & $\sigma$ & $\mu$ & $\sigma$ & $\mu$ & $\sigma$ & $\mu$ & $\sigma$ \\
\hline Case 1 - No Extreme Weather & 5.06 & 1.01 & 71.68 & 19.61 & 13.61 & 3.79 & 84.90 & 107.51 & 5.80 & 1.96 \\
\hline Case 2 - With Extreme Weather & 5.98 & 0.89 & 175.0 & 31.62 & 29.23 & 5.06 & 92.30 & 18.97 & 16.60 & 4.42 \\
\hline
\end{tabular}

Table 5

Results for weather-related failures. Average of 4000 repetitions of the simulations.

\begin{tabular}{|c|c|c|c|c|c|c|c|c|c|c|}
\hline \multirow[t]{2}{*}{ IEEE14 } & \multicolumn{2}{|c|}{$\widehat{\mathrm{ART}}_{i}[\mathrm{~h} / \mathrm{occ}]$} & \multicolumn{2}{|c|}{${\widehat{\mathrm{ART}_{y}}}_{y}[\mathrm{~h} / \mathrm{y}]$} & \multicolumn{2}{|c|}{$\widehat{\mathrm{AFF}}[\mathrm{occ} / \mathrm{y}]$} & \multicolumn{2}{|c|}{$\widehat{\mathrm{ALS}}[\mathrm{MW} / \mathrm{occ}]$} & \multicolumn{2}{|c|}{$\widehat{\mathrm{EENS}}[\mathrm{GW} \mathrm{h}]$} \\
\hline & $\mu$ & $\sigma$ & $\mu$ & $\sigma$ & $\mu$ & $\sigma$ & $\mu$ & $\sigma$ & $\mu$ & $\sigma$ \\
\hline High wind & 6.67 & 1.90 & 99.15 & 25.93 & 14.86 & 3.79 & 103.10 & 95.50 & 10.43 & 3.86 \\
\hline Lighting & 5.32 & 1.77 & 3.89 & 4.43 & 0.73 & 0.70 & 85.60 & 88.54 & 0.33 & 0.57 \\
\hline High wind + lightning & 6.83 & 2.02 & 0.22 & 1.26 & 0.031 & 0.19 & 110.50 & 94.23 & 0.03 & 0.13 \\
\hline
\end{tabular}


Interestingly, in our application we observe a general, significant increase in the estimates of the average reliability/availability indicators when severe weather events are included in the model, as opposed to the results obtained in [16], where a distribution network is analyzed. In fact, even though the duration of extreme weather events is only a small fraction of the grid operating time, the consequences are harsher than in a distribution system, due to the multiplying effect of cascading failures. This behavior is less evident for the indicator $\widehat{\mathrm{ART}}_{i}$ because, as stated above, it is related to the cascading failure consequences only indirectly, through the restoration model. The standard deviations of the reliability/availability indicators, which are important measures of the operator's exposure to risk, are also observed to be higher in case extreme weather events are included in the framework, in agreement with the results obtained in [16].

Note that the proposed framework should be used by the grid operators to (i) check the current grid reliability/availability performances with respect to any thresholds set by the regulatory authorities and (ii) to compare alternative actions to be undertaken, in support of a decision making process involving grid improvements and operation and maintenance strategies. In particular, the different options could be ranked according to the values of the indicators reported in Table 4, possibly assigning different weights to the various indexes according to the final objective of the decision making process. Then, in order to further inform the decision making process, the results of Table 5 could be used to identify the typology of weather event to which the grid is mostly exposed to from a reliability/availability point of view, so as to possibly steer the investments towards more tailored interventions.

The computational time required by the simulations is mainly driven by the number of calls to the cascading failure model. The computational time is approximately $4.5 \mathrm{~h}$ for Case 1 and $10 \mathrm{~h}$ for Case 2, on a $2.26 \mathrm{GHz}$ Intel Core 2 Duo processor with $2 \mathrm{~GB}$ of RAM. In Case 2, the occurrence of extreme weather events leads to a significant increase in the number of line failures, each of which requires a cascading failure simulation. The computational time is expected to increase more than linearly with the size of the grid, as a consequence of the combined effects of (i) the large annual average number of failures, simply due to the long total extension of the lines and (ii) the large computational resources required by a single cascading failure simulation on a more complex network. Rough estimates carried out by running a few cascading failure simulations for the IEEE118 reference network suggest that the proposed analysis would require computational times of the order of days/one week on the same machine. This means that an analysis of a more realistic grid is probably still feasible, although more complex analyses, e.g. including uncertainty and sensitivity analyses or optimizations, would require either to resort to processor clusters, or to identify strategies for accelerating the computations, possibly based on the use of surrogate, approximating models.

\section{Conclusions}

In this work, we have developed a modeling and simulation framework for the quantification of reliability/availability indexes of power transmission grids, which represents a fundamental task for the cost-effective development, operation, maintenance and improvement of these complex infrastructures.

More specifically, the purpose of the proposed framework is that of allowing for a more realistic reliability/availability evaluation, by including a representation of the cascading failure dynamics triggered by extreme weather events and by introducing a new, flexible restoration model which allows to capture the uncertainties related to the repair processes.

The framework has been developed by originally combining stochastic models for describing uncertain weather conditions with a cascading failure model based on a DC approximation of the power flows and a proportional re-dispatch strategy. The novel restoration model accounts for the harsher conditions offered by extreme weather events, under which a repair crew may be forced to operate, thus realistically representing the downtime of a power grid upon a cascading failure event.

The model evaluation has been performed by a customized sequential time Monte Carlo simulation scheme of resolution.

The main assumptions of the proposed framework, introduced in the paper, are here critically revisited:

(a) Only failures of the grid's lines are taken into account. The failures of other grid's components, such as buses, substations, and transformers are not included in the analysis. This is a common approach followed in many works of literature (since power transmission line failures are more common than bus failures [28]) which may lead to an overestimation of the reliability/availability of the grid.

(b) The cascading failure model for the estimation of the line failures' consequences is based on a DC approximation of the power flows. This is also a very popular strategy adopted in both research works and actual applications, especially when the network analyzed is very large, making the AC power flow computations almost infeasible in many practical applications. As recognized in (Kenneth Van den Bergh, Erik Delarue and William D'Haeseleer, DC power flow in unit commitment models, TME Working Paper - Energy and Environment (2014)): "In general, one can conclude that for high voltage grids - which are mostly the ones looked at in unit commitment models - the accuracy of DC power flows is around 5\%, compared to AC power flow and averaged over all lines. The deviation on single line flows can be much larger. Hence, the accuracy of DC power flow is acceptable in the scope of unit commitment models, but one should always keep the limitations of DC power flow in mind and be careful with drawing conclusions about single lines".

Since the purpose of our work is that of analyzing average behaviors and not single line's performances, we consider the DC approximation as the best option, especially in view of a possible application of our framework in optimization or sensitivity problems requiring multiple runs.

Another simplification made in the proposed cascading failure model is that of using a simple, proportional power flow re-dispatch strategy to balance the islands forming in the grid when many of the grid's line are disconnected as the cascading failure proceeds. In general, optimizations techniques are used to re-balance the islands in order to guarantee that the loads are not shed while minimizing the losses. These methods can be easily embedded in the proposed framework, but they require much longer computational times. We chose not to do that in order to be able to perform our research in acceptable times according to the computational power available.

(c) The distributions of the times of occurrence of the severe weather events and of the line failures, and the distributions of the weather event intensities are taken from the works of literature cited in the Introduction. The parameters of the distributions must indeed be identified on the basis of weather statistics collected in the geographical region of interest. 
(d) Also the models relating the intensities of the weather events to the magnitude of the lines' failure rates are taken from previous works of literature, where the relationships were devised on the basis of both physical and empirical reasonings.

Then, the main barriers to the applicability of the method are:

(a) The computational times required for the analysis of large grids with many lines. However, since there are no realtime requirements, this issue can be addressed by investing on an appropriate amount of computational power, if single reliability/availability estimates are required. Optimizations and uncertainty and sensitivity analyses requiring multiple runs of the proposed tool would be more problematic, as already stated at the end of Section 3.

(b) The availability of sufficient weather events statistics in the geographical region of interest, so as to be able to identify the parameters of the distributions used in the weather stochastic model. Usually, weather events databases are available, so that the problem actually reduces to that of properly organizing and treating the data for the parameter identification process.

An application on the well known IEEE14 reference power grid has been given. The results show that rare, but highly severe (in terms of duration and intensity), weather events may have, in general, a significant impact on the distributions of the reliability/ availability performance indicators of a power transmission grid, not only in terms of the standard deviations, as already highlighted in literature for power distribution grids, but also in terms of the means, since their consequences are enhanced by the possible development of cascading failures. Furthermore, in the application considered, it turns out that the duration of the severe weather events has a more significant influence on the reliability/availability of a power grid than their intensity: in fact, a larger failure rate, with prolonged effects on the failure statistics, may result in multiple failures and increased restoration periods, giving rise to larger and long-lasting load losses.

The proposed framework can be used by the grid operators to control and show that the current grid reliability/availability performance levels fulfill the regulatory constraints or to support the decision making on possible grid improvements and different maintenance/restoration strategies, by allowing comparisons and rankings of the available options. In addition to that, the method also automatically provides a simple sensitivity analysis by identifying the typology of weather event to which the grid is mostly exposed to from a reliability/availability point of view, thus further supporting decisions aiming at protecting the grid from the hazards of natural events.

The application has been demonstrated on a small, but realistic, network due to the limited computational power available. The use of the DC approximation for the power flow solution guarantees that sufficiently accurate estimates can be obtained also for larger networks at acceptable computational expenses, at least for comparing a few relevant scenarios. Thorough optimizations and uncertainty/sensitivity analysis, on the other hand, would probably require to include additional approximation schemes in the computational framework, which however would stand valid with no significant modifications.

\section{References}

[1] Eser P, Singh A, Chokani N, Abhari RS. Effect of increased renewables generation on operation of thermal power plants. Appl Energy 2016;164:723-32.
[2] Union for the Co-ordination of transmission of Electricity (UCTE). Final report of the investigation committee on the 28 September 2003 Blackout in Italy: 2004.

[3] U.S.-Canada Power System Outage Task Force. Final report on the August 14, 2003 blackout in the United States and Canada: causes and recommendations; 2004.

[4] Union for the Co-ordination of transmission of Electricity (UCTE). Final Report System Disturbance on November, 4 2006. Technical report; 2007.

[5] Sanstad AH, McMenamin S, Sukenik A, Barbose GL, Goldman CA. Modeling an aggressive energy-efficiency scenario in long-range load forecasting for electric power transmission planning. Appl Energy 2014;128:265-76.

[6] Lin J, Sun Y-Z, Cheng L, Gao W-Z. Assessment of the power reduction of wind farms under extreme wind condition by a high resolution simulation model. Appl Energy 2012;96:21-32.

[7] Kezunovic M, Dobson I, Dong Y. Impact of extreme weather on power system blackouts and forced outages: new challenges. In: Balkan power conference, Šibenik Croatia, September 2008.

[8] Campbell RJ. Weather-related power outages and electric system resiliency. In: Congressional research service report for congress. Available: <https:// www.fas.org/sgp/crs/misc/R42696.pdf>.

[9] Billinton R, Allan RN. Reliability evaluation of power systems. New York: Plenum; 1984.

[10] Billinton R, Wu C, Singh G. Extreme adverse weather modeling in transmission and distribution system reliability evaluation. In: 14th PSCC, Sevilla, Spain, 2428 June.

[11] Alvehag K, Söder L. A stochastic weather dependent reliability model for distribution systems. In: Presented at the probabilistic methods applied to power systems, Rincon, Puerto Rico; 2008.

[12] Brown RE, Gupta S, Christie RD, Venkata SS, Fletcher R. Distribution system reliability assessment: momentary interruptions and storms. IEEE Trans Power Delivery 1997;12(4):1569-74.

[13] Lallemand C. Methodology for risk based asset management M.Sc. dissertation. Stockholm (Sweden): School Elect. Eng., Royal Inst. Technol. (KTH); 2008.

[14] Balijepalli N, Venkata SS, Richter Jr CW, Christie RD, Longo VJ. Distribution system reliability assessment due to lightning storms. IEEE Trans Power Delivery 2005;20(3):2153-9.

[15] Zhou Y, Pahwa A, Yang S. Modeling weather-related failures of overhead distribution lines. IEEE Trans Power Syst 2006:21(4):1683-90.

[16] Alvehag K, Söder L. A reliability model for distribution systems incorporating seasonal variations in severe weather. IEEE Trans Power Delivery 2011;26 (2):910-9. art. no. 5669368

[17] Li G, Zhang P, Luh PB, Li W, Bie Z, Serna C, Zhao Z. Risk analysis for distribution systems in the northeast U.S. under wind storms. IEEE Trans Power Syst 2014;29(2):889-98. art. no. 6654346.

[18] Dueñas-Osorio L, Vemuru SM. Cascading failures in complex infrastructure systems. Struct Saf 2009;31(2):157-67.

[19] Zimmerman RD, Murillo Sánchez CE, Thomas RJ. MATPOWER: “Steady-State Operations, Planning, and Analysis Tools for Power Systems Research and Education". IEEE Trans Power Syst 2011;26(1):12-9.

[20] Dobson I, Carreras Ba, Lynch VE, Newman DE. An initial model for complex dynamics in electric power system blackouts. In: Proc 34th Annu Hawaii Int Conf Syst Sci.

[21] Christie RD. Power systems test case archive. Department of Electrical Engineering, University of Washington; 1999. Available: <http://www.ee. washington.edu/research/pstcai>.

[22] Motter AE, Lai YC. Cascade-based attacks on complex networks. Phys Rev E Stat Nonlinear Soft Matter Phys 2002;66:2-5.

[23] Rosas-Casals M, Valverde S, Solé RV. Topological vulnerability of the European power grid under errors and attacks. Int J Bifurc Chaos 2007; $17: 2465-75$

[24] Buldyrev SV, Parshani R, Paul G, Stanley HE, Havlin S. Catastrophic cascade of failures in interdependent networks. Nature 2010;464:1025-8.

[25] Zio E, Sansavini G. Component criticality in failure cascade processes of network systems. Risk Anal 2011;31:1196-210.

[26] Crucitti P, Latora V, Marchiori M. Model for cascading failures in complex networks. Phys Rev E: Stat, Nonlin, Soft Matter Phys 2004;69:045104.

[27] Carreras B, Lynch VE, Dobson I, Newman DE. Critical points and transitions in an electric power transmission model for cascading failure blackouts. Chaos 2002;12:985-94.

[28] Andersson G et al. Causes of the 2003 major grid blackouts in North America Europe, and recommended means to improve system dynamic performance. IEEE Trans Power Syst 2005;20:1922-8.

[29] Rocchetta R, Li YF, Zio E. Risk assessment and risk-cost optimization of distributed power generation systems considering extreme weather conditions. Reliab Eng Syst Safe 2015;136:47-61.

[30] Tapper M. 2003-2007 Yearly outage statistics reports from DARWin. Available: <http://www.svenskenergi.se/sv/Vi-ar-betar-med/Nat/ Drifthandelsestatistik-DARWin/>.

[31] Sweden before climate changes-treats and possibilities. Swedish Government, no. SOU 2007:60; 2007.

[32] Cadini F, Azzolin A, Zio E. Cascading failure behavior in randomly generated power transmission networks. In: ESREL - Safety and Reliability of Complex Engineered Systems, 1825-1832, ISBN 978-1-138-02879-1. Zurich, Switzerland, September 7-10 2015. 
[33] Bienstock D. Optimal control of cascading power grid failures. In: Proc IEEE Conf Decis Control. p. 2166-73.

[34] Wood Allen J, Wollenberg BF. Power generation operation and control, 2nd ed. In: John Wiley\&Sons, Inc., WILEY-INTERSCIENCE.

[35] Galbusera L, Theodoridis G, Giannopoulos G. Model predictive control of energy transmission grids accounting for power-ICT interdependencies. In: 22nd Mediterranean Conference on Control and Automation (MED), University of Palermo, June 2014, Palermo, Italy.

[36] Zio E, Sansavini G. Vulnerability of smart grids with variable generation and consumption: a system of systems perspective. IEEE Trans Syst Man Cybern A Syst Humans 2013;43:477-87.
[37] Allan RN, Billinton R, Sjarief I, Goel L, So KS. A reliability test system for educational purposes-basic distribution system data and results. IEEE Trans Power Syst 1991;6(2):813-20.

[38] Grisby LL. Power systems. CRC Press; 2007.

[39] Zio E, Piccinelli R. Randomized flow model and centrality measure for electrical power transmission network analysis. Reliab Eng Syst Safe 2010;95(4):379-85.

[40] IEEE Transaction on Power Systems, 14 (3); 1999.

[41] Billinton R, Li W. Reliability assessment of electric power system using Monte Carlo Methods. New York: Plenum Press; 1994. p. 19-20. 